\title{
Some properties of $\boldsymbol{k}$-neighborhood Template $\boldsymbol{A}$-type two-dimensional bounded cellular acceptors
}

\author{
Makoto Nagatomo*, Makoto Sakamoto, Hikaru Susaki, Tuo Zhang, \\ Satoshi Ikeda and Hiroshi Furutani \\ Faculty of Engineering, University of Miyazaki, Miyazaki 889-2192, Japan \\ Tsutomu Ito, Takao Ito \\ Institute of Engineering, Hiroshima University, Higashi-Hiroshima, Hiroshima 739-8527, Japan \\ Yasuo Uchida \\ Department of Business Administration, Ube National College of Technology, Ube, Yamaguchi 755-8555, Japan
}

Tsunehiro Yoshinaga

Department of Computer Science and Electronic Engineering, Tokuyama college of Technology, Shunan, Yamaguchi 745-8585, Japan

*Corresponding author, E-mail: sakamoto@cs.miyazaki-u.ac.jp

Tel: +81-985-58-7392, Fax: +81-985-58-7392

\begin{abstract}
In this paper, we investigate multi-dimensional computational model, k-neighborhood template $A$-type three-dimensional bounded cellular acceptor on four-dimensional tapes, and discuss some basic properties. This model consists of a pair of a converter and a configuration-reader. The former converts the given four-dimensional tape to three-dimensional configuration. The latter determines whether or not the derived three-dimensional configuration is accepted, and concludes the acceptance or non-acceptance of given four-dimensional tape. We mainly investigate some open problems about $k$-neighborhood template $A$-type three-dimensional bounded cellular acceptor on four-dimensional tapes whose configuration-readers are $L(m)$ space-bounded deterministic (nondeterministic) three-dimensional Turing machines.
\end{abstract}

Keywords: configuration-reader, converter, four-dimension, neighbor, space-bounded, Turing machine

\section{Introduction}

Due to the advances in many application areas such as computer animation, dynamic image processing, and so on, the study of four-dimensional pattern processing has been of crucial importance. Thus, the study of four-dimensional automata as the computational models of four-dimensional pattern processing has been meaningful. From this point of view, we first proposed four-dimensional automata as computational models of four-dimensional pattern processing in 2002, and investigated their several accepting powers[2]. By the way, in the multi-dimensional pattern processing, designers often use a strategy whereby features are extracted by projecting high-dimensional space on low-dimensional space. So, from this viewpoint, we introduce a new computational model, $k$-neighborhood template $A$-type three-dimensional bounded cellular acceptor (abbreviated as $A-3 B C A(k)$ ) on four-dimensional tapes, and discuss some basic properties[3]. An $A-3 B C A(k)$ consists of a pair of a converter and a configuration-reader. The former converts the given four-dimensional tape to three-dimensional configuration. The latter determines 
whether or not the derived three-dimensional configuration is accepted, and concludes the acceptance or non-acceptance of given four-dimensional tape. When an input four-dimensional tape is presented to the $A-3 B C A(k)$, a three-dimensional cellular automaton as the converter first reads it to the future direction at unit speed (i.e., one three-dimensional rectangular array per unit time). From this process, the four-dimensional tape is converted to a configuration of the converter which is a state matrix of a three-dimensional cellular automaton. Second, three-dimensional automaton as the configuration-reader reads the configuration and determines its acceptance. We say that an input four-dimensional tape is accepted by the $A-3 B C A(k)$ if and only if the configuration is accepted by the configuration-reader. Therefore, the accepting power of the $A-3 B C A(k)$ depends on how to combine the converter and the configuration-reader. An $A-3 D B C A(k)$ $(A-3 N B C A(k))$ is called a $k$-neighborhood template $A$-type three-dimensional deterministic bounded cellular acceptor ( $k$-neighborhood template $A$-type three-dimensional nondeterministic bounded cellular acceptor). This paper mainly investigates some open problems about accepting powers of $A-3 D B C A(k)$ 's whose configuration-readers are $L(m)$ space-bounded deterministic (nondeterministic) three-dimensional Turing machines[1].

\section{Preliminaries}

Definition 2.1. Let $\Sigma$ be a finite set of symbols. A three-dimensional tape over $\Sigma$ is a four-dimensional rectangular array of elements of $\Sigma$. The set of all four-dimensional tapes over $\Sigma$ is denoted by $\Sigma(4)$. Given a tape $x \in \Sigma(4)$, for each integer $\mathrm{j}(1 \leq j \leq 4)$, we let $m_{j}(x)$ be the length of $x$ along the $j$-th axis. The set of all $x \in \Sigma$ with $l_{1}(x)=m_{1}, l_{2}(x)=m_{2}, l_{3}(x)=m_{3}$, and $l_{4}(x)=m_{4}$ denoted by $\sum_{1}^{\left(\mathrm{m}_{1}, \mathrm{~m}_{2}, \mathrm{~m}_{3}, \mathrm{~m}_{4}\right)}$. If $1 \leq i_{j} \leq l j(x)$ for each $j(1 \leq j \leq 4)$, let $x\left(i_{1}, i_{2}, i_{3}, i_{4}\right)$ denote the symbol in $x$ with coordinates $\left(i_{1}, i_{2}, i_{3}, i_{4}\right)$. Furthermore, we define $x\left[\left(i_{1}, i_{2}, i_{3}, i_{4}\right),\left(i_{1}{ }^{\prime}, i_{2}{ }^{\prime}, i_{3}{ }^{\prime}, i_{4}{ }^{\prime}\right)\right]$, when $1 \leq i_{j} \leq i^{\prime}{ }_{j} \leq l_{j}(x)$ for each integer $j(1 \leq j \leq 4)$, as the four-dimensional tape $\mathrm{y}$ satisfying the following (i) and (ii):

(i) for each $j(1 \leq \mathrm{j} \leq 4), l_{j}(y)=i_{j}{ }_{j}-i_{j}+1$;

(ii) for each $\mathrm{r}_{1}, \mathrm{r}_{2}, \mathrm{r}_{3}, \mathrm{r}_{4}\left(1 \leq r_{1} \leq l_{1}(y), 1 \leq r_{2} \leq l_{2}(y)\right.$, $\left.1 \leq r_{3} \leq l_{3}(y), 1 \leq r_{4} \leq l_{4}(y)\right), y\left(r_{1}, r_{2}, r_{3}, r_{4}\right)=$ $x\left(r_{1}+i_{1}-1, r_{2}+i_{2}-1, r_{3}+i_{3}-1, r_{4}+i_{4}-1\right)$. (We call $x\left[\left(i_{1}, i_{2}, i_{3}, i_{4}\right),\left(i_{1}, i_{1}{ }_{2}, i_{3}, i^{\prime}{ }_{4}\right)\right] x\left[\left(i_{1}, i_{2}, i_{3}, i_{4}\right)\right.$, $\left.\left(i_{1}, i_{2}, i_{3}, i_{4}\right)\right]$-segment of $x$.);
We now introduce a $k$-neighborhood template $A$-type three-dimensional bounded cellular acceptor $(A-3 \mathrm{BCA}(k))$, which is a main object of discussion in this paper.

Definition 2.2. Let $A$ be the class of an automaton moving on a three-dimensional configuration. Then, an $A$-3BCA $(k) M$ is defined by the 2-tuple $M=(R, B) . R$ and $B$ are said to be a converter and a configuration-reader in view of its property, respectively.

(1) $R$ is a three-dimensional infinite array consists of the same finite state machines and is defined by the 6-tulpe $\quad M=\left(\mathrm{Z}^{2}, \mathrm{~N}^{2}, \mathrm{~K}, \Sigma, \sigma, \mathrm{q}_{0}\right)$, where

(1) $Z$ is the set of all integer, and the finite state machines are assigned to each point of $Z^{3}(=Z x Z x Z)$. The finite state machine situated at coordinates $(i, j, k) \in Z^{3}$ is called the $(i, j, k)$-th cell and denoted by $\mathrm{A}(i, j, k)$,

(2) $\mathrm{N}^{3}\left(\subseteq \mathrm{Z}^{3}\right)$ represents the neighborhood template of each cell and $\mathrm{N}^{3}=\{(i, j, k)$ $-1 \leq i, j, k \leq 1\}$,

(3) $\mathrm{K}$ is a finite set of states of each cell and contains $q_{\#}$ (the boundary state) and $q_{0}$ (the initial state),

(4) $\Sigma$ is a finite set of input symbols (\#£ $\Sigma$ is the boundary symbol),

(5) $\sigma: \mathrm{K}^{27} \times\left(\sum \cup\{\#\}\right) \rightarrow 2^{\mathrm{K}}$ is the cell state transition function. Let $q_{1 . j, k}(t)$ be the state of the $A(i, j, k)$ at time $t$. Then

$q_{i, j, k}(t+1) \in \sigma\left(q_{i-1, j-1, k-1}(t), \quad q_{i-1, j, k-1}(t), \quad q_{i-1, j+1, k-1}(t)\right.$, $q_{i, j-1, k-1}(t), \quad q_{i, j, k-1}(t), \quad q_{i, j+1, k-1}(t), \quad q_{i+1, j-1, k-1}(t), \quad q_{i+1, j, k-1}(t)$, $q_{i+1, j+1, k-1}(t), \quad q_{i-1, j-1, k}(t), \quad q_{i-1, j, k}(t), \quad q_{i-1, j+1, k}(t), \quad q_{i, j-1, k}(t)$, $q_{i, j, k}(t), \quad q_{i, j+1, k}(t), \quad q_{i+1, j-1, k}(t), \quad q_{i+1, j, k}(t), \quad q_{i+1, j+1, k}(t)$, $q_{i-1, j-1, k+1}(t), q_{i-1, j, k+1}(t), q_{i-1, j+1, k+1}(t), q_{i, j-1, k+1}(t), q_{i, j, k+1}(t)$, $\left.q_{i, j+1, k+1}(t), q_{i+1, j-1, k+1}(t), q_{i+1, j, k+1}(t), q_{i+1, j+1, k+1}(t), a\right)$, wher $a$ is the symbol on the $A(i, j, k)$ at time $t$. If $q_{i, j, k}(t)=$ $q_{\#}$, however, $q_{i, j, k}(t+1)=\left\{q_{\#}\right\}$ for each $(i, j, k) \in \mathrm{Z}^{3}$ and each $t \geq 0$.

(2) A set of input symbols of $B$ is $\mathrm{K}-\left\{q_{\#}\right\}$ (where $B$ $\in A)$. Intuitively, $M=(R, B)$ moves as follows, given a four-dimensional input tape $x \in \sum^{(m 1, m 2, m 3, m 4)}$ $\left(m_{1}, m_{2}, m_{3}, m_{4} \geq 1\right) \quad(x$ is surrounded by the boundary symbol \#). First, each cell $A(i, j, k)$ of $R\left(1 \leq i \leq m_{1}, 1 \leq j \leq m_{2}, 1 \leq k \leq m_{3}\right)$ reads each symbol on the first three-dimensional rectangular array $x(i, j, k, 1)$ in the initial state $q_{0}$, and all of the other cells read the boundary symbols \#'s in the boundary state $q_{\#}$ 's at time $t=0$. Starting from this 
condition, $R$ keeps reading $x$ according to the cell state transition function, and moving down the cell array by one three-dimensional rectangular array, every time $R$ reads one three-dimensional rectangular array all. Next, $B$ starts to move regarding a three-dimensional configuration of $R$ just after $R$ finished reading $x$ as a three-dimensional input tape and determines whether or not can accept the configuration. If $B$ accepts it, $x$ is said to be accepted by $M$. Let $T(M)$ be the set of all accepted three-dimensional tape by M.

Definition 2.3. An $A-3 B C A$ in Dfinition 2.2 is called a 27 -neighborhood template $A-3 B C A$. If we deal with east, west, south, north, up, down neighboring cells and remarkable cell, we call it 7-neighborhood template $A-3 B C A$. If we deal with only remarkable cell, we call it 1-neighborhood template $A-3 B C A$. From now on, we denote k-neighborhood template $A-3 B C A$ by $A-3 B C A(\mathrm{k})$ $(k \in\{1,7,27\})$.

Definition 2.4. If the image generated by $\sigma$ in Definitions 2.2 and 2.3 is a singleton, the converter is said to be deterministic, and if not, it is said to be nondeterministic. An $A-3 B C A(k)(k \in\{1,7,27\})$, which converter is deterministic (nondeterministic), is said to be a deterministic (nondeterministic). $A-3 B C A(k)$ and denoted by $A-3 D B C A(k)(A-3 N B C A(k))$.

We now consider the class of three-dimensional automata described by the following abbreviations as the class of the configuration-reader of $A-3 B C A(k) A$. In this paper, we assume that the reader is familiar with the definition of these automata. If necessary, see[2].

3-DTM $(L(m)) \cdots$ The class of $L(m)$ space-bounded deterministic three-dimensional Turing machine $3-N T M(L(m)) \quad \cdots$ The class of $L(m)$ space-bounded nondeterministic three-dimensional Turing machine

$D O \cdots$ The class of deterministic three-dimensional on-line tessellation acceptor

$$
D B \quad \cdots \quad \text { The class of }
$$

deterministic two-dimensional bounded cellular acceptor

For example 3-DTM(L(m))-3DBCA(27) represents such the class as its converter is deterministic and 27 -neighborhood, and its configuration-reader is an $L(m)$ space-bounded deterministic three-dimensional Turing machine. Moreover, for any $A \in\{3-D T M(L(m))$,
3-NTM(L(m)) $\}$, for any $X \in\{D, N\}$ and for any $k \in\{1,7$, $27\}$, the class of set of all four-dimensional tapes accepted by $A-3 X B C A(k)$ is denoted by $\mathrm{L}[A-3 X B C A(k)]$. We let each side-length of each input tape of these automata be equivalent in order to increase the theoretical interest.

\section{Main results}

In this section, we discuss some properties of $A-3 B C A(k)$ 's whose configuration-readers are $L(m)$ space-bounded deterministic (nondeterministic) three-dimensional Turing machines.

First, we show that a relationship between determinism and nondeterminism, when we use $L(m)$ space-bounded three-dimensional Turing machines for any $L(m)>\mathrm{m}^{3}$ as the configuration-readers.

Theorem 3.1. For
$\mathcal{L}\left[3-\mathrm{XTM}\left(\mathrm{m}^{3}\right)-3 \mathrm{DBCA}(1)\right]=$$\quad \begin{array}{rl}\mathcal{L} & X\end{array}$ [3-XTM $\left.\left(\mathrm{m}^{3}\right)-3 \mathrm{NBCA}(1)\right]$.

Proof: From above definition, it is obvious that $\mathcal{L}\left[3-X T M\left(m^{3}\right)-3 D B C A(1)\right]$ $\mathcal{L}\left[3-X T M\left(m^{3}\right)-3 D B C A(1)\right]$. We below show that $\mathcal{L}\left[3-\mathrm{XTM}^{\mathrm{s}}\left(\mathrm{m}^{3}\right)-3 \mathrm{NBCA}(1)\right] \subseteq$ $\mathcal{L}\left[3-\mathrm{DTM}\left(\mathrm{m}^{3}\right)-3 \operatorname{DBCA}(1)\right]$ (we can prove another case (i. e. , $X=N)$ in the same way).

Now, let $M=(R, B)\left(\left(R=\mathrm{Z}^{2}, \mathrm{~N}^{2}, \mathrm{~K}, \quad \Sigma, \sigma, \mathrm{q}_{0}\right), \quad B=(\mathrm{K}\right.$, $\left.\left.\mathrm{Q}, \quad \Sigma, \Gamma, \delta, \mathrm{p}_{0}, \mathrm{~F}\right)\right)$ be some 3-DTM( $\left.m^{3}\right)-3 N B C A(1)$. Then, we consider an $M^{\prime}=\left(R^{\prime}, B^{\prime}\right)\left(R^{\prime}=\left(\mathrm{Z}^{3}, \mathrm{~N}^{3}, \mathrm{~K}, \quad \Sigma\right.\right.$, $\left.\left.\sigma^{\prime}, \mathrm{q}_{0}\right), B^{\prime}=\left(\mathrm{K}^{\prime}, \mathrm{Q}, \Sigma, \Gamma, \delta^{\prime}, \mathrm{p}_{0}^{\prime}, \mathrm{F}^{\prime}\right)\right)$ constructed as follows.

(1) Construction of $R^{\prime}$

(1) $\mathrm{K}^{\prime}=2^{\left(\mathrm{K}-\left\{\mathrm{q}_{\#}\right\}\right)} \cup\left\{\mathrm{q}_{\#}\right\}, \mathrm{q}_{0}^{\prime}=\left\{\mathrm{q}_{0}\right\}$.

(2) For any $\mathrm{a} \in \Sigma$ and any $\mathrm{K}^{\prime \prime} \in \mathrm{K}^{\prime}-\left\{\mathrm{q}_{\#}\right\}$,

(2) Construction of $B^{\prime}$ $\sigma^{\prime}\left(\mathrm{K}^{\prime},, \mathrm{a}\right)=\bigcup_{q \in K^{\prime \prime}} \sigma(\mathrm{p}, \mathrm{a})$.

For any $p \in \mathrm{Q}$ and any $\mathrm{K}^{\prime \prime} \in \mathrm{K}^{\prime}-\left\{\mathrm{q}_{\#}\right\}$, $\delta^{\prime}\left(p, \mathrm{~K}^{\prime \prime}\right)=\bigcup_{r \in K^{\prime \prime}} \delta(p, r)$.

Intuitively, we explain the movement of $M^{\prime}=\left(R^{\prime}, B^{\prime}\right)$ constructed in this way. Let us suppose that a four-dimensional tape $x \in \Sigma^{(4)^{+}}$is given to $\mathrm{M}^{\prime}$. R' is one-neighborhood, so we can consider each cell of R' as usual one-dimensional finite automata. Then, each cell of $R$ ' moves to store all states that each corresponding cell of $R$ can enter in each state at each time by using the well-known subset construction method (see (1)).

$B$ ' nondeterministically chooses only one state from each state of each cell of $R^{\prime}$, and simulates the 
movement of $B$ regarding the selected states as the input symbol. If $B^{\prime}$ can not accept the input, $B$ ' selects the next input and simulates the movement of $B$. From the way such as the above manner, $B^{\prime}$ checks the all input patterns, and if $B^{\prime}$ can accept one input, $B^{\prime}$ can accept the configuration of $R^{\prime}$ (see (2)).

It is clear that $T\left(M^{\prime}\right)=T(M)$ for $M^{\prime}=\left(R^{\prime}, B^{\prime}\right)$ constructed in this manner.

Next, we show that there exists a language accepted by a 3-DTM(0)-3NBCA(1), but not accepted by any 3-NTM(L(m))-3DBCA(27) for any $L(m))=\mathrm{o}(\log m)$.

Theorem 3.2. For any function $L(m)=\mathrm{o}(\ell \circ g \quad m)$, $\mathcal{L}[3-\mathrm{DTM}(0)-3 \mathrm{NBCA}(1)]-\mathcal{L}[3-\mathrm{NTM}(\mathrm{L}(\mathrm{m}))-3 \mathrm{DBCA}(27)$ ]$\neq \phi$.

Proof: Let $\mathrm{C}=\left\{\mathrm{w}_{0} 2 \mathrm{w}_{1} 2 \cdots 2 \mathrm{w}_{\mathrm{k}} \mid k \geq 1 \& \forall i(0 \leq i \leq k)\right.$ $\left.\left[\mathrm{w}_{1} \in\{0,1\}^{+}\right] \&{ }^{\exists} j(0 \leq j \leq k)\left[\mathrm{w}_{0}=\mathrm{w}_{j}{ }^{\mathrm{r}}\right]\right\}$ (where, for any one-dimensional tape $\mathrm{w}, \mathrm{w}^{\mathrm{r}}$ denotes the reversal of $\left.\mathrm{w}\right)$, and $T_{1}=\left\{x \in\{0,1,2\}{ }^{(4)+} \mid \exists \mathrm{m}>3\left[\ell_{1}(x)=\ell_{2}(x)=\ell_{3}(x)=\ell_{4}(x)\right.\right.$ $=\mathrm{m} \& x[(1,1, m, m),(1, m, m, m)] \in \mathrm{C}]\}$. Then, by using a technique similar to that in the proof of Lemma 2(1) in [4], we can show that $T_{1}$ is accepted by 3-DTM(0)-3NBCA(1), but not accepted by any 3-NTM( $L(m))-3 D B C A(27)$ for any $L(m))=\mathrm{o}(\log m)$.

Corollary 3.1. For any $L(m)=\mathrm{o}(\log m)$ and any $X \in$ $\{D, N\}, \quad \mathcal{L}[3-X T M(L(m))-3 D B C A(1)]$

$\mathcal{L}[3-X T M(L(m))-3 N B C A(1)]$.

Remark 3.1. By using a technique to that in the proof of Theorem 3 in [4], we can show that for any function $L(m)$ and any $k \in\{7,27\}, \mathcal{L}[3-X T M(L(m))-3 D B C A(k)]$ $\subsetneq \mathcal{L}[3-X T M(L(m))-3 N B C A(k)]$.

Finally, by using the well-known technique, we can show that there exists a language accepted by a $D O-3 N B C A(1)$ and a $D B-3 N B C A(1)$, but not accepted by any $3-D T M(\mathrm{~L}(m))-3 D B C A(27)$ for any function $L$ $(m)=\mathrm{o}(\log m)$.

Theorem 3.3. For any function $L(m)=\mathrm{o}(\log m),(\mathcal{L}$ $[D O-3 N B C A(1)] \quad \cap \quad \mathcal{L}[D B-3 N B C A(1)])-$ $\mathcal{L}[3-D T M(L(m))-3 D B C A(27)] \neq \phi$.

\section{Conclusion}

We conclude this paper by giving the following problem. For any $X \in\{D, N\}$ and any $L(m)(\log m \leq$ $L(m)$ and $\left.L(m)=\mathrm{o}\left(m^{2}\right)\right), \mathcal{L}[X T M(L(m))-3 D B C A(1)] \subsetneq$ $\mathcal{L}[X T M(L(m))-3 N B C A(1)] ?$

\section{References}

1. M. Sakamoto, H. Okabe, and K. Inoue, "Some properties of four-dimensional finite automata", 2002 Chugoku-Section Joint Convention Record of Institutes of Electrical and Information Engineering, Shimane, Japan (2002), p.351.

2. M. Sakamoto, "Three-dimensional alternating Turing machines", Ph.D. Thesis, Yamaguchi University (1999).

3. M. Sakamoto, M. Nagatomo, X. Feng, T. Kurogi, T. Zhang, T. Ito, Y. Uchida, T. Yoshinaga, S. Ikeda, M. Yokomichi, and H. Furutani, "Hierarchy based on k-neighborhood template about k-neighborhood template A-type three-dimensional bounded cellular acceptor", Proceedings of the International Conference on Artificial Life and Robotics (ICAROB2014), Compal Hall, Oita, Japan, OS9-1 (2014)

4. H. Taniguchi, K. Inoue, and I. Takanami, "k-neighborhood template A-type 2-dimensional bounded cellular acceptor", IECE of Japan Transactions (D), Vol.69, No.3 (1986), pp.291-301. 